\title{
Factores de riesgo de enfermedades crónicas no transmisibles en funcionarios de una empresa de servicios financieros de la Región Metropolitana
}

\author{
María del Pilar Fagalde ${ }^{1}$, José Antonio del Solar $\mathbf{H}^{2,3}$, \\ Marcia G uerrero B ${ }^{2 a}$, Eduardo Atalah $\mathbf{S}^{4}$. \\ Risk factors for non communicable \\ chronic diseases among workers \\ of a financial company
}

Background: The epidemic of cardiovascular diseases in Chile, requires the development of strategies in health promotion and prevention. Aim: To assess the prevalence of risk factors for chronic non communicable diseases among workers of a financial company in Metropolitan Santiago. Material and Methods: Assessment of 2,225 workers (1,383 males with a median age of 49 years and 842 females with a median age of 43 years). All answered an enquiry about education, medical history, smoking habits and physical activity. Body mass index and blood pressure were measured and a blood sample was obtained to measure blood glucose and lipid levels. Logistic repression models were used to determine the main risk factors for hypertension, diabetes, obesity, hypercholesterolemia and hyperuricemia. Results: Sixteen percent of studied subjects were obese, $49 \%$ had overweight, $57 \%$ had hypercholesterolemia, $28 \%$ had high blood pressure, $4 \%$ were diabetic, $4 \%$ had hyperuricemia, $45 \%$ smoked and $83 \%$ were sedentary. Each worker had a mean of $2.4 \pm 1.1$ risk factors. This figure was significantly higher among men, obese subjects, those older than 40 years and those with a lower educational level. Conclusions: There is an important disease burden among the studied subjects, specially among obese and older individuals. Healthy lifestyles should be promoted in this population (Rev Méd Chile 2005; 133: 919-28).

(Key Words: Cardiovascular diseases; Environment and Public Health; Risk factors)

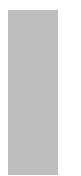

\footnotetext{
Recibido el 4 de noviembre, 2004. Aceptado el 13 de abril, 2005.

${ }^{1}$ Unidad Asistencia Nutricional Intensiva, Hospital DIPRECA. ${ }^{2}$ Mutual de Seguridad, Santiago. ${ }^{3}$ Clínica Las Condes, Santiago. ${ }^{4}$ Departamento de Nutrición, Facultad de Medicina, Universidad de Chile.

aEnfermera Universitaria.
}

Correspondencia a: Eduardo Atalah S. Independencia 1027, Santiago, Chile. Fax: 737-8778. Fono: 678-6239. E mail: eatalah@med.uchile.cl 
L as enfermedades crónicas no transmisibles (ECNT) constituyen un problema creciente a nivel mundial. Este fenómeno se atribuye a los cambios que han experimentado la mayor parte de los países, entre los que destaca el control de las enfermedades infecciosas, el aumento de las expectativas de vida y la adquisición progresiva de un modelo de vida occidental $\gg, 2$. Chile no ha estado ajeno a esta situación y muestra en la actualidad un perfil epidemiológico semejante al de países con mayor desarrollo, con un predominio de ECNT, tales como enfermedades cardiovasculares, cerebrovasculares, cánceres, diabetes mellitus, obesidad y accidentes ${ }^{3-8}$.

Diversos factores de riesgo intervienen en la evolución de estas enfermedades, muchos de los cuales están asociados a estilos de vida, hábitos y costumbres que introducen la modernización, tales como sedentarismo, dietas inadecuadas, estrés, tabaquismo, consumo de alcohol y drogas. Los daños atribuibles a estos factores se pueden disminuir al aplicar técnicas propias de la Medicina del Trabajo, interfiriendo oportunamente en la historia natural de ellos. El desarrollo de medidas preventivas será de mayor utilidad, cuanto más se conozca de la historia natural de estas enfermedades y del comportamiento de los factores de riesgo asociados ${ }^{2,9}$. La baja percepción sobre estas patologías por parte de la población es otra barrera importante, que a través de la Salud Ocupacional se podría lograr un mayor y efectivo control ${ }^{10}$.

En nuestro país diversos estudios han puesto en evidencia la alta prevalencia de ECNT y sus factores de riesgo en la población general adulta. Entre ellos destaca el estudio CARMEN ${ }^{5}$, la Encuesta Nacional de Calidad de Vida y Salud del Ministerio de Salud ${ }^{10}$ y más recientemente la Encuesta Nacional de Salud, realizada por la Pontificia Universidad Católica ${ }^{11}$. Estas enfermedades determinan un alto costo personal, familiar y social. Son responsables de significativas pérdidas económicas relacionadas al costo directo de cuidados médicos así como al costo indirecto derivado de la pérdida de productividad ${ }^{12-17}$. Su conocimiento es especialmente importante en personas económicamente activas, grupo en el cual existen mayores facilidades para realizar una intervención por tratarse de una población de más fácil acceso.
El objetivo del trabajo fue medir los factores de riesgo de ECNT en un grupo de adultos laboralmente activos, con el fin de planificar una estrategia de intervención. Se eligió una empresa de servicios financieros de la Región Metropolitana con aproximadamente 3.570 funcionarios, con gran estabilidad laboral (18 años promedio de permanencia), donde $94 \%$ de ellos está afiliado a una misma ISAPRE. El propósito final es proponer acciones preventivas específicas para esta población, que permitan reducir la incidencia y gravedad de las ECNT con un esquema de seguimiento del tipo Programa Riesgo Persona que integra estas patologías comunes con los demás riesgos laborales.

\section{Material y MÉTodo}

Se analizó una base de datos de más de 13.000 personas de 25 empresas previamente evaluadas con el Programa Riesgo Persona de la Mutual de Seguridad de la Cámara Chilena de la Construcción. Seleccionamos, para el estudio, una empresa de servicios financieros por tener una cobertura de exámenes superior al $80 \%$. En esa institución se ofreció un control de salud estandarizado y gratuito a todos los funcionarios (3.578) asegurando la confidencialidad de la información, de los cuales $2.915(81,4 \%)$ aceptaron voluntariamente ser sometidos al estudio.

Previo aviso cada funcionario fue visitado en su lugar de trabajo por una enfermera universitaria entrenada, quien les dio a conocer el objetivo del estudio, instrucciones para responder un formulario auto administrado y el requisito de obtener una muestra de sangre previo ayuno nocturno de al menos $8 \mathrm{~h}$. La confidencialidad de la información fue crucial al garantizar que los datos serían guardados en la Mutual de Seguridad y que la Empresa sólo recibiría los resultados globales sistematizados para compararse con otras empresas o definir la posibilidad de intervenir de manera informada sobre las variables de mayor riesgo.

En los días siguientes, los participantes fueron entrevistados nuevamente para revisar los cuestionarios y aclarar dudas o completar las preguntas sin contestar. Simultáneamente se midió la talla, peso y se obtuvo la presión arterial en condicio- 
nes estandarizadas. Finalmente se citó a cada paciente para examen médico y entrega de los resultados.

La encuesta incluía: datos demográficos (edad, sexo, estado civil, nivel educacional y actividad laboral) y antecedentes autorreportados sobre hipertensión arterial, dislipidemias, diabetes, tabaquismo y actividad física. El nivel educacional fue clasificado en 4 categorías: básica o media incompleta, media completa, técnica o universitaria incompleta y universitaria completa. El tabaquismo se clasificó en: nunca ha fumado, fumador actual y ex fumador. Se consideró físicamente activa personas que realizaban como mínimo ejercicio durante media hora al menos tres veces por semana o sedentario aquellos que no realizaban ninguna actividad física o por un tiempo o frecuencia menor ${ }^{11}$.

El peso se obtuvo con ropa ligera y descalzo con una balanza eléctrica marca Tanita, calibrada periódicamente y la talla con un podómetro portátil, siguiendo las normas del Ministerio de Salud $^{18}$. Se calculó el Indice de Masa Corporal (IMC) (peso/talla ${ }^{2}$ y se consideró enflaquecido $<18,5$; normal 18,5 a 24,9; sobrepeso 25,0 a 29,9; obesidad $>30$.

La presión arterial se obtuvo previo reposo de al menos 10 min, mediante un esfigmomanómetro digital OMRON, modelo HEM-601. En caso de una presión sistólica $\geq 140$ o diastólica $\geq 90 \mathrm{mmHg}$, se repitió la medición a los 10 min y se registró el promedio de ambas determinaciones. Se consideró hipertensión arterial una presión sistólica $\geq 140$ mmHg o una presión diastólica $\geq 90 \mathrm{mmHg}^{19}$.

Previo ayuno nocturno, se tomó una muestra de sangre de $8 \mathrm{ml}$ en una habitación previamente habilitada en cada sucursal de la empresa. Se utilizaron dos tubos marca Greiner, uno de $3 \mathrm{ml}$ para medición de glicemia y otro de $5 \mathrm{ml}$ para perfil bioquímico. La muestra fue trasladada refrigerada a $4^{\circ} \mathrm{C}$, dentro de las $5 \mathrm{~h}$ siguientes, a un laboratorio privado, donde fueron procesadas. El colesterol y la glicemia se determinaron con reactivos Boehringer Manheim, utilizando la técnica enzimática colorimétrica GOD-PAP y un equipo autoanalizador automatizado marca Dave. La glicemia fue clasificada según los criterios para estudios epidemiológicos de American Diabetes Association $^{20}$ : $<110 \mathrm{mg} / \mathrm{dl}$ normal; $>110<126$ intolerancia a glucosa, $>126$ probable diabetes mellitus. Se consideró colesterol total elevado niveles $\geq 200 \mathrm{mg} / \mathrm{dl}$, de acuerdo a los criterios del Programa Nacional de Educación del Colesterol en Estados Unidos de Norteamérica (USA) ${ }^{21}$.

Se calculó el número de factores de riesgo presentes en cada persona, considerando hipertensión arterial, hipercolesterolemia, tabaquismo, diabetes, sedentarismo, hiperuricemia y obesidad $\mathrm{y}$, posteriormente, se estimó el promedio $\pm \mathrm{DE}$ en función del sexo, edad, estado nutricional y nivel educacional.

Estadística. Los datos fueron procesados con el programa STATA 6.0 analizándose en primer lugar la normalidad de cada variable. Dado que la mayor parte de las variables continuas no tuvieron una distribución normal (excepto el número de factores de riesgo), se utilizó la distribución percentilar y los intervalos de confianza como medida de tendencia central y el test de Kruskal Wallis para la comparación entre grupos. En las variables nominales y categóricas, se analizó distribución de frecuencia y $\chi^{2}$. Por medio de regresiones logísticas se determinaron los modelos que mejor explicaban las patologías estudiadas controlando el efecto de variables de confusión. Las variables de exposición fueron analizadas en forma dicotómica, considerando como factor de riesgo las siguientes categorías: edad mayor de 40 años, sexo masculino, sedentarismo, IMC $\geq 30 \mathrm{y}$ escolaridad básica o media. En una segunda etapa se realizó un análisis de regresión logística multifactorial con el procedimiento de adición de variables una a una, considerando como criterio de inclusión una probabilidad de 0,05 y de eliminación de 0,2. El modelo propuesto permite estudiar el efecto conjunto de la exposición a distintos factores, controlando el efecto de variables de confusión. En todos los casos se consideró significativas diferencias con un valor de $\mathrm{p}<0,05$.

\section{RESULTADOS}

La muestra final quedó constituida por 2.225 personas (62\% hombres y $38 \%$ mujeres), que representan a $62,2 \%$ de la población total de la institución. Seiscientos noventa funcionarios fueron excluidos del análisis por no realizarse los exámenes de sangre solicitados. El mayor porcen- 
taje de la población tenía educación media completa (42\%) o estudios técnicos o universitarios incompletos (30\%). La distribución de esta variable fue más favorable en mujeres $(p<0,001)$ y en menores de 40 años (datos no mostrados).

La mediana y sus intervalos de confianza de las principales variables estudiadas se presentan en la Tabla 1. El grupo tenía una mediana de edad de 46 años (95\% IC 46-47) y una estatura superior a lo observado en sectores de menores ingresos (171 y $160 \mathrm{~cm}$ en hombres y mujeres, respectivamente). En hombres destacó una mediana de colesterol plasmático e IMC superior al límite normal y valores significativamente mayo- res que las mujeres en todas las variables ( $p$ $<0,001)$. El 35\% de la población tenía un estado nutricional normal y el resto sobrepeso (49\%) u obesidad (16\%). Sólo tres personas presentaron bajo peso $(0,4 \%)$, por lo que no fueron consideradas en los análisis posteriores. La prevalencia de exceso de peso aumentaba con la edad en ambos sexos y fue significativamente mayor en los hombres $(p<0,01)$. En los menores de 40 años, más de la mitad de la población ya tenía exceso de peso.

La prevalencia de cada uno de los factores de riesgo estudiados se muestra en la Tabla 2. El sedentarismo fue altamente prevalente, seguido

Tabla 1. Mediana y $95 \%$ intervalos de confianza de las principales variables estudiadas, según sexo

\begin{tabular}{|lccc|}
\hline Variables & $\begin{array}{c}\text { Hombres } \\
\mathrm{n}=1.380\end{array}$ & $\begin{array}{c}\text { Mujeres } \\
\mathrm{n}=845 \\
\mathrm{p} 50(95 \% \mathrm{IC})\end{array}$ & $\mathrm{p}(95 \% \mathrm{IC})$ \\
\hline Edad años & $49(48-49)$ & $43(41-45)$ & \\
Peso kilos & $79(78-80)$ & $64(63-64)$ & $<0,001$ \\
Talla cm & $171(170-171)$ & $160(159-160)$ & \\
IMC & $27(26,7-27,2)$ & $24,8(24,5-25,1)$ & $<0,001$ \\
Presión sistólica mmHg & $128(127-130)$ & $119(117-120)$ & $<0,001$ \\
Presión diastólica mmHg & $80(80-81)$ & $77(76-78)$ & $<0,001$ \\
Colesterol mg/dl & $211(208-213)$ & $200(196-204)$ & $<0,001$ \\
Glicemia mg/dl & $85(84-86)$ & $79(78-79)$ & $<0,001$ \\
Uricemia mg/dl & $5,0(4,9-5,0)$ & $3,4(3,3-3,5)$ & $<0,001$ \\
\hline
\end{tabular}

Tabla 2. Prevalencia y promedio de factores de riesgo según sexo

\begin{tabular}{|lcccc|}
\hline Factores de riesgo & $\begin{array}{c}\text { Hombres } \\
\mathrm{n}=1.380 \\
\%\end{array}$ & $\begin{array}{c}\text { Mujeres } \\
\mathrm{n}=845 \\
\%\end{array}$ & $\begin{array}{c}\text { Total } \\
\mathrm{n}=2.225 \\
\%\end{array}$ & $\mathrm{p}$ \\
\hline Obesidad & 18,6 & 12,5 & 16,3 & $<0,01$ \\
Hipertensión arterial & 35,9 & 16,2 & 28,5 & $<0,01$ \\
Hipercolesterolemia & 61,7 & 50,0 & 57,2 & $<0,01$ \\
Diabetes & 5,2 & 1,5 & 3,8 & $<0,01$ \\
Hiperuricemia & 6,5 & 1,2 & 4,5 & $<0,01$ \\
Tabaquismo & 42,7 & 49,5 & 45,3 & $<0,01$ \\
Sedentarismo $_{\text {Promedio }}^{*} \pm$ DE & 79,8 & 86,9 & 82,5 & $<0,01$ \\
\end{tabular}

*Factores de riesgo de enfermedades crónicas. 
por la hipercolesterolemia y el tabaquismo. En menor proporción se encuentra la hipertensión y obesidad. Se encontraron diferencias significativas por sexo en todas las variables, con una prevalencia casi 3 veces mayor de hipertensión arterial, diabetes e hiperuricemia en hombres. El tabaquismo fue el único factor de riesgo más frecuente en mujeres, aunque los datos disponibles no permiten discriminar el número de cigarrillos diarios. El promedio global de enfermedades crónicas 0 factores de riesgo fue $2,5 \pm 1,2$ y $2,2 \pm 1,0$ para hombres y mujeres, respectivamente. Sólo 3\% de la población estudiada no presentó ningún factor de riesgo y la gran mayoría presentó entre 2 y 4 .

La prevalencia de casi todas las variables aumentó significativamente con la edad (Tabla 3), especialmente diabetes, hipertensión y obesidad. El sobrepeso alcanzó un máximo en el grupo mayor de 50 años, superando el 75\% al considerar en forma conjunta los valores de IMC $\geq 25$ (datos no mostrados). Destaca además un alto porcentaje de población joven con hipercolesterolemia. El tabaquismo fue el único factor de riesgo que disminuía a lo largo de la vida. El promedio de factores de riesgo en todas las edades es mayor a dos, produciéndose un aumento significativo después de los 40 años ( $\mathrm{p}<0,01$ ).

La prevalencia de hipertensión arterial, diabetes e hiperuricemia prácticamente se duplica con el sobrepeso y se triplica con la obesidad (Tabla 4), lo que se refleja también en el promedio de factores de riesgo de enfermedades crónicas. No

Tabla 3. Prevalencia y promedio de factores de riesgo según edad

\begin{tabular}{|lccccc|}
\hline Factores de riesgo & $\begin{array}{c}20-39 \text { años } \\
\mathrm{n}=759 \\
\%\end{array}$ & $\begin{array}{c}40-49 \text { años } \\
\mathrm{n}=554 \\
\%\end{array}$ & $\begin{array}{c}50-59 \text { años } \\
\mathrm{n}=817 \\
\%\end{array}$ & $\begin{array}{c}60-70 \text { años } \\
\mathrm{n}=95 \\
\%\end{array}$ & $\mathrm{p}$ \\
\hline Obesidad & 11,2 & 15,2 & 20,7 & 26,3 & $<0,01$ \\
Hipertensión arterial & 12,5 & 25,9 & 42,5 & 50,5 & $<0,01$ \\
Hipercolesterolemia & 43,8 & 62,4 & 65,9 & 60,6 & $<0,01$ \\
Diabetes & 0,7 & 3,3 & 5,7 & 17,0 & $<0,01$ \\
Hiperuricemia & 3,7 & 4,3 & 5,2 & 5,3 & $\mathrm{NS}$ \\
Tabaquismo & 52,8 & 53,3 & 36,4 & 16,8 & $<0,01$ \\
Sedentarismo $_{\text {Promedio* }}^{*}$ DE & 83,0 & 81,6 & 83,6 & 74,7 & $\mathrm{NS}$ \\
\end{tabular}

*Factores de riesgo de enfermedades crónicas.

Tabla 4. Prevalencia y promedio de factores de riesgo según estado nutricional

\begin{tabular}{|lcccc|}
\hline Factores de riesgo & $\begin{array}{c}\text { Normal } \\
\mathrm{n}=770\end{array}$ & $\begin{array}{c}\text { Sobrepeso } \\
\mathrm{n}=1.092\end{array}$ & $\begin{array}{c}\text { Obeso } \\
\mathrm{n}=363\end{array}$ & $\mathrm{p}$ \\
\hline Hipertensión arterial & 14,6 & 30,8 & 51,2 & $<0,01$ \\
Hipercolesterolemia & 48,7 & 61,8 & 61,8 & $<0,01$ \\
Diabetes & 2,1 & 3,4 & 7,2 & $<0,01$ \\
Hiperuricemia & 2,0 & 5,2 & 7,8 & $<0,01$ \\
Tabaquismo & 46,9 & 45,4 & 41,3 & $\mathrm{NS}$ \\
Sedentarismo $^{*}$ & 83,5 & 80,7 & 86,2 & $\mathrm{NS}$ \\
Promedio $^{*} \mathrm{DE}$ & $2,0 \pm 0,9$ & $2,3 \pm 1,0$ & $3,6 \pm 1,1$ & $<0,01$ \\
\hline
\end{tabular}

* Factores de riesgo de enfermedades crónicas. 
hubo diferencias, en cambio, en el tabaquismo y sedentarismo según esta variable.

El nivel de escolaridad mostró una relación inversa con la prevalencia de obesidad, hipertensión y diabetes, lo que se traduce en un mayor promedio de factores de riesgo de enfermedades crónicas en el grupo con educación básica y media incompleta (Tabla 5).

La importancia relativa de las diferentes variables estudiadas en la prevalencia de enfermedades crónicas, según modelos de regresión logística multivariados se presenta en la Tabla 6. La edad mayor a 40 años y el sexo masculino son las variables que más se repiten como factor de riesgo en las distintas patologías. El sedentarismo se suma como factor de riesgo para hipertensión y obesidad y la obesidad también se incluye como un factor de riesgo independiente para hipertensión y diabetes mellitus. Aun cuando todas las variables seleccionadas en los modelos son estadísticamente significativas, explican una proporción relativamente baja de la varianza, especialmente para la obesidad, hipercolesterolemia e hiperuricemia.

\section{DisCUSIÓN}

En el análisis e interpretación de los resultados se debe considerar que el grupo estudiado no es una muestra representativa de la población, dado que se trata de personas laboralmente activas, dentro del concepto de medicina laboral de trabajador sano, lo que no permite extrapolar este estudio a la población general. Esta aparente debilidad del diseño podría considerarse también una fortaleza, ya que en Chile existe poca información en este importante segmento poblacional ${ }^{22}$.

La muestra final quedó constituida por aquellos funcionarios que espontáneamente aceptaron someterse al estudio y que finalmente se realizaron los exámenes de laboratorio en condiciones estandarizadas (62\% del universo). Este hecho podría determinar un sesgo de selección, por la probabilidad de que las personas con mayores antecedentes de las patologías estudiadas estuvieran más motivadas a participar. También se podría argumentar en forma inversa, en el sentido que este grupo pudo marginarse al tener antecedentes sobre su situación de salud. Es difícil responder si efectivamente hubo un sesgo, aunque al comparar el sexo y edad del grupo estudiado con el universo de la institución no se encontraron diferencias significativas, lo que nos permite suponer que la muestra fue representativa. Otro elemento en contra de un sesgo de selección es que una proporción importante de las personas detectadas con hipertensión y diabetes, desconocían esta condición.

Tabla 5. Prevalencia y promedio de factores de riesgo según nivel educacional

\begin{tabular}{|lccccc|}
\hline Factores de riesgo & $\begin{array}{c}\text { Básica/media } \\
\text { incompleta } \\
\mathrm{n}=129 \\
\%\end{array}$ & $\begin{array}{c}\text { Media } \\
\text { completa } \\
\mathrm{n}=895 \\
\%\end{array}$ & $\begin{array}{c}\text { Técnica/Univ } \\
\text { Incompleta } \\
\mathrm{n}=647 \\
\%\end{array}$ & $\begin{array}{c}\text { Universitaria } \\
\mathrm{n}=475 \\
\%\end{array}$ & $\mathrm{p}$ \\
\hline Obesidad & 33,3 & 16,6 & 15,2 & 13,0 & $<0,01$ \\
Hipertensión arterial & 45,7 & 30,7 & 22,6 & 27,2 & $<0,01$ \\
Hipercolesterolemia & 66,9 & 61,4 & 49,9 & 57,8 & $<0,01$ \\
Diabetes & 12,6 & 4,5 & 2,6 & 1,5 & $<0,01$ \\
Hiperuricemia & 7,1 & 5,0 & 3,3 & 4,0 & $<0,01$ \\
Tabaquismo & 42,6 & 44,7 & 50,0 & 41,1 & $\mathrm{NS}$ \\
Sedentarismo $_{\text {Promedio* }}+\mathrm{DE}$ & 84,5 & 82,2 & 82,5 & 81,5 & $\mathrm{NS}$ \\
\end{tabular}

${ }^{*}$ Factores de riesgo de enfermedades crónicas. 
Tabla 6. Razón de disparidad (O R) para el riesgo de cada una de las patologías estudiadas, estimadas mediante modelos de regresión logística multivariado

\begin{tabular}{|lccc|}
\hline Factor de riesgo & OR & $95 \% \mathrm{CI}$ & $\mathrm{p}$ \\
\hline Hipertensión arterial & & & \\
Edad $>40$ años & 3,6 & $2,8-4,6$ & $<0,001$ \\
Sexo masculino & 2,6 & $2,1-3,3$ & $<0,001$ \\
Sedentarismo & 1,7 & $1,3-2,2$ & $<0,001$ \\
Obesidad & 2,9 & $2,3-3,7$ & $<0,001$ \\
$\mathrm{R}^{2}=12,3 \%$ & & & \\
Diabetes & & & \\
Edad $>40$ años & 5,8 & $2,3-14,7$ & $<0,001$ \\
Sexo masculino & 2,6 & $1,4-4,8$ & $<0,001$ \\
Obesidad & 1,9 & $1,1-3,1$ & $<0,001$ \\
Escolaridad (básica y & 1,8 & $1,1-2,9$ & \\
media) & & & $<0,001$ \\
$\mathrm{R}^{2}=9,2 \%$ & & & $<0,001$ \\
Obesidad & & & $<0,001$ \\
Edad $>40$ años & 1,8 & $1,3-2,3$ & \\
Sexo masculino & 1,5 & $1,2-1,9$ & $<0,001$ \\
Sedentarismo & $1,0-2,0$ & $<0,001$ \\
$\mathrm{R}^{2}=2,0 \%$ & & & $<0,001$ \\
Hipercolesterolemia & & & \\
Edad $>40$ años & & $1,8-2,6$ & \\
Sexo masculino & $1,2-1,7$ & \\
Sedentarismo & $1,0-1,6$ & \\
$\mathrm{R}^{2}=3,5 \%$ & & & \\
Hiperuricemia & & $2,9-11,1$ & \\
Sexo masculino & & & \\
$\mathrm{R}^{2}=5,0 \%$ & 1,5 & & \\
\hline
\end{tabular}

Se observó una alta prevalencia de factores de riesgo de enfermedades crónicas no transmisibles, los que ya están presentes en proporciones importantes en adultos jóvenes. Esta situación es especialmente preocupante considerando que éstos actuarán por períodos prolongados, produciendo un deterioro progresivo en la salud, de no tomarse medidas de prevención o control.

En este estudio la prevalencia de obesidad y sobrepeso fue mayor en hombres que en mujeres, a diferencia de lo encontrado en estudios nacionales previos ${ }^{4,5,11}$. Esto podría explicarse en parte por el mayor nivel educacional de las mujeres estudiadas, variable que se asocia con una menor prevalencia de obesidad ${ }^{11,25}$. Eventualmente podría explicarse por algún grado de discriminación laboral en función de la apariencia personal, considerando que se trata de una empresa de servicios.

Setenta y cinco por ciento de los hombres y $48 \%$ de las mujeres presentaban exceso de peso (obesidad más sobrepeso), lo que se podría explicar por el efecto conjunto del sedentarismo y conductas alimentarias inadecuadas, caracteriza- 
das por dietas con una alta densidad calórica, ricas en ácidos grasos saturados, azúcar y un bajo consumo de verduras, frutas, granos integrales y otros alimentos ricos en fibra ${ }^{24}$. Sería de interés completar el estudio con el análisis de la alimentación, considerando que esta población recibe el almuerzo en un casino institucional y sería posible realizar una intervención a ese nivel.

De los diferentes factores de riesgo estudiados el más prevalente es el sedentarismo y es más bien la norma de conducta habitual, como se ha demostrado en otros estudios nacionales, no mostrando diferencias importantes en función del estado nutricional o el nivel educacional. Este factor debe priorizarse como intervención por su favorable relación costo-beneficio, con efectos positivos sobre la obesidad, hipertensión arterial, diabetes, hipercolesterolemia, osteoporosis y calidad de vida.

Prácticamente un tercio de la población estudiada era hipertensa, proporción que superó el $50 \%$ en los obesos y mayores de 60 años. La prevalencia de hipertensión se duplicó con el sobrepeso y triplicó con la obesidad. El sexo masculino también fue un factor de riesgo de esta patología. La presencia de hipertensión en mujeres fue prácticamente la mitad a la observada en la encuesta nacional de 2003, lo que podría explicarse por el mayor nivel educacional de nuestra población, variable que se asocia inversamente con esta patología.

La hipercolesterolemia afectó casi al 40\% de los menores de 40 años, situación similar a lo que se ha encontrado en otros estudios nacionales ${ }^{5,26}$ y en un reciente estudio realizado en funcionarios de empresas de servicios ${ }^{22}$. También observamos que a menor nivel educacional aumentaba la prevalencia de hipercolesterolemia, situación diferente a lo encontrado en la encuesta nacional, donde el nivel educacional no tuvo relación con esta patología.

El tabaquismo fue más frecuente en mujeres, al igual que lo publicado por Lanas y $\operatorname{cols}^{22}$, pero distinto a los estudios realizados en población general. En la Encuesta Nacional de Salud la prevalencia de fumadores fue mayor en hombres, aunque las mujeres con más años de estudio fumaban más que los hombres de su mismo estrato, situación que podría explicar lo que ocurre en las funcionarias de esta institución. Al igual que en otros estudios, observamos mayor frecuencia de tabaquismo en las personas más jóvenes.

Del total de personas diagnosticadas como diabéticas o hipertensas en el estudio $82 \%$ y $67 \%$, respectivamente no tenía diagnóstico previo (datos no mostrados). Con esto se confirma la importancia de realizar evaluaciones preventivas periódicas de las características de la vigilancia epidemiológica de Mutual de Seguridad en los riesgos laborales, considerando que un tratamiento oportuno permite reducir las complicaciones y una muerte prematura. Es fundamental además educar a la población para que tome conciencia de los riesgos de estas patologías, factores predisponentes y conozca y aplique las medidas de prevención correspondientes.

Con este estudio queda demostrado, una vez más, la urgente necesidad de integración de la salud común y la salud laboral y desarrollar programas multidisciplinarios para enfrentar esta nueva epidemia de factores de riesgo de enfermedades crónicas no transmisibles. Es necesario que todos los sectores participen activamente (educación, salud, sector privado, organismos no gubernamentales, etc), para lograr prevenir 0 revertir la situación actual. En el caso de empresas, como la estudiada, debe aprovecharse la situación en que es una spoblación cautiva», donde cualquier tipo de intervención o seguimiento se facilita.

Dentro de las actividades propuestas se puede destacar: informar a los funcionarios de los resultados obtenidos; actividades educativas para promover estilos de vida saludables que involucren tanto a los funcionarios como a sus familias; seguimiento y control de aquellos individuos de mayor riesgo, a través de un programa de control de enfermedades crónicas no transmisibles; incentivar en los casinos el consumo de dietas más saludables, incluyendo información nutricional de los alimentos, mayor aporte de verduras y frutas y menor aporte de calorías, grasas y sal; estimular la actividad física en la vida cotidiana (uso de escaleras, instalación de un gimnasio en la misma institución o convenio con alguno cerca); desarrollar programas para dejar de fumar y definir políticas institucionales al respecto, como prohibir fumar en oficinas y espacios comunes. Estas 
medidas pueden contribuir a mejorar la calidad de vida de esta población y a reducir la pesada carga

\section{REFERENCIAS}

1. Popkin BM. An overview on the nutrition transition and its health implications: the Bellagio meeting. Public Health Nutrition 2002; 5: 93-103.

2. KenNeth G. The global impact of noncommunicable diseases: estimates and projections. World Health Stat Q, 41 (1998).

3. Albala C, Vio F, Kain J, Uauy R. Nutrition transition in Chile: determinants and consequences. Public Health Nutrition 2002; 5: 123-8.

4. Berríos $\mathrm{X}$, Koponen $\mathrm{T}$, Huiguang $\mathrm{T}$, Khaltaev N, PusKa P, Nissinen A. Distribution and prevalence of major risk factors of noncommunicable diseases in selected countries: the WHO Inter- Health Programme. Bulletin of the World Health Organization 1997; 75: 99-108.

5. Jadue L, Vega J, Escobar MC, Delgado I, Garrido C, LASTRA P ET AL. Factores de riesgo para las enfermedades no transmisibles: Metodología y resultados globales de la encuesta de base del programa CARMEN (Conjunto de Acciones para la Reducción Multifactorial de las Enfermedades no Transmisibles). Rev Méd Chile 1999; 127: 100413.

6. Vio F, Albala C, Crovetto M. Promoción de salud en la transición epidemiológica de Chile. Rev Chil Nutr 2000; 27: 21-9.

7. Albala C, Vio F, Kain J, Uauy R. Nutrition transition in Latin America: the case of Chile. Nutrition Reviews 2001; 59: 170-6.

8. Albala C, Kain J, Burrows R, Díaz E (Eds). Obesidad, un desafío pendiente. Imprenta Salesianos, Santiago 2000.

9. WHO. Obesity, Preventing and Managing the Global Epidemic. Report of a WHO Consultation of Obesity. Geneva, 3-5 June 1997.

10. Ministerio de Salud. Encuesta Nacional de Calidad de Vida y Salud. Departamento de epidemiología y promoción de salud, Ministerio de Salud, Santiago, 2001.

11. Ministerio de Salud. Encuesta Nacional de Salud 2003, Santiago, marzo 2004. http://www.minsal.cl/ de enfermedad que enfrentará en un futuro no muy lejano.

ici/destacados/Folleto\%20FINALpdf consultado el 4 de junio de 2004).

12. British Cardiac Society, British Hyperlipidaemia Association, British Hypertension Society, British Diabetic Association. Joint British recommendations on prevention of coronary heart disease in clinical practice: summary. BMJ 2000; 320: 705-8.

13. YANG MC, HuANG IC. Establishing a cost estimation model for hypertension and its related diseases in Taiwan. J Formos Med Assoc 1999; 98: 394-402.

14. Kisskinen U, Vartiainen E, Puska P, Aromaa A. Longterm cost and life-expectancy consequences of hypertension. J Hypertens 1998; 16: 1103-12.

15. Wolf AM, Colditz GA. Current estimates of the economic cost of obesity in the United States. Obes Res 1998; 6: 97-106.

16. Levy E, Levy P, Le Pen C, Basdevant A. The economic cost of obesity: the French situation. Int J Obes Relat Metab Disord 1995; 19: 788-92.

17. TuCKER LA, FRIEDMEN GM. Obesity and Absenteeism: an epidemiologic study of 10.825 employed adults. Am J Health Promot 1998; 12: 202-7.

18. Programa de Salud del Adulto: Examen de Salud Preventivo del Adulto", Ministerio de Salud, Santiago, Julio 1995.

19. The six report of the national committee on prevention, detection, evaluation, and treatment of high blood pressure. Arch Int Med 1997; 157: 2413-46.

20. Expert Committee on Diagnosis and Classification of Diabetes Mellitus. Report of the Expert Committee on Diagnosis and Classification of Diabetes Mellitus. Diabetes Care 2000; 23 (Supp 1): 54-5.

21. Executive Summary of the Third Report of National Cholesterol Education Program (NCEP) Expert Panel on Detection, Evaluation, and Treatment of High Blood Cholesterol in Adults (Adult Treatment Panel III). JAMA 2001; 285: 2486-97.

22. Lanas F, Del Solar JA, Maldonado M, Guerrero M, Espinoza F. Prevalencia de Factores de Riesgo de Enfermedad Cardiovascular en una Población de 
Empleados Chilenos. Rev Méd Chile 2003; 131: 129-34.

23. Filozof C, González C, Sereday M, Mazza C, BraGUINSKY J. Obesity prevalence and trends in LatinAmerican countries. Obes Rev 2001; 2: 99-106.

24. Uauy R, Albala C, Kain Julana. Obesity Trends in Latin America: Transiting from Under- to Overweight. J Nutr 2001; 131: 893-9.
25. Berríos X, Jadue L, Zenteno J, Ross MI, RodrígueZ H. Prevalencia de factores de riesgo para enfermedades crónicas. Un estudio en la población general de la Región Metropolitana. Rev Méd Chile 1990; 118: 597-604.

26. Berríos X, Jadue L, Pierotic M. Perfil lipídico en la población adulta de la Región Metropolitana. Rev Méd Chile 1992; 120: 331-3.

Agradecimientos

Se agradece al Dr. Alejandro Morales, Gerente de Salud Ocupacional de Mutual de Seguridad, por su valiosa colaboración e importantes sugerencias en la elaboración de este manuscrito. 